\title{
Fratura toracolombar explosão: correlação entre o comprometimento do canal vertebral e os resultados do tratamento conservador
}

\author{
Thoracolumbar burst fracture: correlation between the spinal canal \\ compromise and the clinical results of conservative treatment \\ Fractura toralumbar por explosión: correlación entre el \\ comprometimiento del canal vertebral y resultados del tratamiento \\ conservador
}

\author{
Osmar Avanzi \\ Elcio Landin ${ }^{2}$ \\ Robert Meves ${ }^{3}$ \\ Maria Fernanda Silber Caffaro 4 \\ Juliano De Bortoli ${ }^{5}$
}

\section{RESUMO}

Introdução: o tratamento das fraturas explosão toracolombares sem comprometimento neurológico é controverso. A porcentagem de estreitamento do canal vertebral tem sido utilizada como parâmetro de indicação cirúrgica, mas a sua significância em pacientes sem comprometimento neurológico permanece incerta. Objetivo: avaliar pacientes com fratura toracolombar explosão, correlacionando o estreitamento inicial do canal vertebral e os resultados clínicos do tratamento conservador. Métodos: foram avaliados de forma retrospectiva os prontuários, radiografias e filmes de tomografia computadorizada

\section{ABSTRACT}

Introduction: there has been considerable controversy regarding what constitutes the best treatment for lumbar burst fractures without neurological compromise. The percentage of spinal canal compromise has been used as a parameter of surgical indication, but its significance in patients without neurological deficit remains uncertain. objective: the purpose of this study was to evaluate patients with thoracolumbar burst fractures correlating the initial percentage of spinal canal compromise and the clinical results of the conservative treatment. Methods: we have retrospectivelyevaluated the clinical

\section{RESUMEN}

Introductión: el tratamiento de las fracturas por explosión toracolumbares sin comprometimiento neurológico es controvertido. El porcentaje de estrechamiento del canal vertebral ha sido utilizado como parámetro de indicación quirúrgica, pero su importancia en pacientes sin compromiso neurológico permanece incierta. Objetivo: el objetivo de este estudio es evaluar pacientes con fractura toracolumbar por explosión, correlacionando el estrechamiento inicial del canal vertebral y los resultados clínicos del tratamiento conservador. Métodos: fueron evaluados de forma retrospectiva las historias clínicas, ra-

\footnotetext{
Trabalho realizado no Departamento de Ortopedia e Traumatologia da Faculdade de Ciências Médicas da Santa Casa de Misericórdia de São Paulo - FCMSCMSP - São Paulo (SP), Brasil.

'Doutor, Professor Adjunto e Consultor do Grupo de Coluna do Departamento de Ortopedia e Traumatologia da Faculdade de Ciências Médicas da Santa Casa de Misericórdia de São Paulo - FCMSCMSP - São Paulo (SP), Brasil.

${ }^{2}$ Doutor, Professor Assistente e Chefe do Grupo de Coluna do Departamento de Ortopedia e Traumatologia da Faculdade de Ciências Médicas da Santa Casa de Misericórdia de São Paulo - FCMSCMSP - São Paulo (SP), Brasil.

${ }^{3}$ Doutor, Professor Assistente do Grupo de Coluna Departamento de Ortopedia e Traumatologia da Faculdade de Ciências Médicas da Santa Casa de Misericórdia de São Paulo - FCMSCMSP - São Paulo (SP), Brasil.

${ }^{4}$ Mestre, Professora Instrutora Assistente do Grupo de Coluna do Departamento de Ortopedia e Traumatologia da Faculdade de Ciências Médicas da Santa Casa de Misericórdia de São Paulo - FCMSCMSP - São Paulo (SP), Brasil.

${ }^{5}$ Médico do Curso de Aperfeiçoamento do Grupo de Coluna do Departamento de Ortopedia e Traumatologia da Faculdade de Ciências Médicas da Santa Casa de Misericórdia de São Paulo - FCMSCMSP - São Paulo (SP), Brasil.
} 
(TC) de pacientes adultos, portadores de fratura toracolombar explosão, incluindo os níveis de T11 a L2, do tipo A3 da classificação de Magerl, com menos de 10 dias de evolução, tratados conservadoramente com o uso de órtese tipo TLSO (Jewett) ou gesso em hiperextensão. Foi ainda aplicado um questionário que incluía as escalas visual- analógico de dor ou EVA, escala de Dor e Trabalho de Denis, índice de Oswestry e questionário de qualidade de vida SF-36. Resultados: não houve correlação entre o estreitamento canal vertebral e a EVA ou a Escala de Dor de Denis. Houve correlação negativa entre a porcentagem de estenose do canal vertebral e a Escala de Trabalho de Denis. Também houve correlação negativa entre o índice de Oswestry e a porcentagem de estenose do canal vertebral, indicando que os pacientes com maiores escores de incapacidade apresentaram menor porcentage de estenose. Na análise de correlação entre os índices obtidos nos domínios do SF-36 e a porcentagem de estenose do canal vertebral, houve correlação significativa apenas no domínio capacidade funcional, indicando que os pacientes com melhor capacidade funcional ou escores mais próximos a 100\%, também apresentavam maior porcentagem de estenose. Conclusão: os resultados obtidos confirmam a inexistência de correlação entre maior estreitamento do canal vertebral e piores resultados clínicos, conforme descrito na literatura.

DESCRITORES: Fraturas da coluna vertebral; Estenose espinhal; Canal vertebral; Vértebras torácicas; Vértebras lombares records, radiographs and CT scans of adult patients with thoracolumbar burst fractures including the levels of T11 to L2, A3 type of Magerl's classification with less than 10 days of evolution, submitted to conservative treatment with TLSO (Jewett) or hyperextension plaster. A questionnaire was also applied including the Visual Analog Pain Scale (VAS), Denis Work and Pain Scale, Oswestry Index and the Quality of Life assessment questionnaire SF36. Results: it was not found correlation between the percentage of spinal compromise and the VAS or the Denis pain scale. Negative correlation was found between the percentage of spinal canal compromise and the Denis work scale. Negative correlation was also found between the Oswestry index and the percentage of spinal canal compromise, indicating that patients with higher disability scores presented less percentage of spinal canal stenosis. In the correlation analysis between the SF-36 values and the percentage of spinal canal stenosis, significant correlation was found only with the Physical Functioning domain, suggesting that the patients with best physical function also presented higher percentage of stenosis. Conclusion: the obtained results confirm the absence of correlation between higher percentages of spinal canal compromise and worse clinical results as described in literature.

KEYWORDS: Spinal fractures; Spinal stenosis; Spinal canal; Thoracic vertebrae; Lumbar vertebrae diografias y videos de Tomografia Computarizada (TC) de pacientes adultos, portadores de fractura toracolumbar por explosión, incluyendo los niveles de T11 a L2, del tipo A3 de la clasificación de Magerl, con menos de 10 días de evolución, tratados de forma conservadora con el uso de ortesis tipo TLSO (Jewett) o yeso en hiperextensión. Fue aun aplicado un cuestionario que incluyó las escalas visual-analógica de dolor o VAS, escala de Dolor y Trabajo de Denis, indice de Oswestry y cuestionario de cualidad de vida SF-36. Resultados: No hubo correlación entre el estrechamiento del canal vertebral y la VAS o la Escala de Dolor de Denis. También hubo correlación negativa entre el índice de Oswestry y el porcentaje de estenosis del canal vertebral, indicando que los pacientes con mayores índices de incapacidad presentaron menor porcentaje de estenosis. En el análisis de correlación entre los índices obtenidos en los dominios de SF-36 y el porcentaje de estenosis del canal vertebral hubo correlación significativa en apenas el dominio Capacidad Funcional, indicando que los pacientes con mejor capacidad funcional o índices más próximos a $100 \%$, también presentaron mayor porcentaje de estenosis.Conclusión: Los resultados obtenidos confirman la inexistencia de una correlación entre el mayor estrechamiento del canal vertebral y los peores resultados clínicos, conforme descrito en la literatura.

DESCRIPTORES: Fracturas de la columna vertebral; Estenosis espinal; Canal vertebral; Vértebras torácicas; Vértebras lumbares

\section{INTRODUÇÃO}

A região toracolombar é o sítio mais comum de fraturas vertebrais. Essas fraturas ocorrem com mais freqüência devido a traumatismos de alta energia e acometem jovens com idade entre 15 e 29 anos do sexo masculinol. De acordo com a definição de Denis2, a fratura toracolombar explosão compromete as colunas anterior e média do corpo vertebral, com a presença de fragmentos ósseos no interior do canal vertebral, levando a estreitamento em graus variáveis. Estas lesões podem resultar em instabilidade mecânica e neurológica, conferindo assim maior ou menor gravidade das lesões ${ }^{2,3}$.

Ao longo dos últimos 50 anos, a controvérsia a respeito do melhor tratamento para esse tipo de fratura se mantém, com estudos sustentando tanto o tratamento cirúrgico quanto a abordagem conservadora, especialmente nos casos sem lesão neurológica ${ }^{4-10}$. 
A porcentagem de estreitamento do canal vertebral tem sido usada como parâmetro para a indicação de tratamento cirúrgico, mas a sua correlação com o comprometimento neurológico ainda é tema de discussão ${ }^{11-16}$. Há diversos trabalhos que correlacionam o estreitamento do canal vertebral com déficit neurológico, mas a sua significância em pacientes sem lesão neurológica permanece incerta $^{17-21}$. Alguns estudos mostram ainda que a reabsorção dos fragmentos ósseos e a remodelação do canal vertebral ocorrem tanto nos pacientes tratados de forma cirúrgica quanto conservadora ${ }^{22-28}$.

O objetivo deste trabalho é avaliar pacientes com fratura toracolombar explosão, correlacionando o estreitamento inicial do canal vertebral e resultados clínicos após o tratamento conservador.

\section{MÉTODOS}

Foram avaliados de forma retrospectiva os prontuários, radiografias e filmes de tomografia computadorizada (TC) de pacientes adultos, portadores de fratura toracolombar explosão, incluindo os níveis de T11 a L2 ${ }^{29}$, do tipo A3 da classificação de Magerl ${ }^{30}$, com menos de dez dias de evolução, tratados de modo conservador pelo grupo de Coluna do Departamento de Ortopedia e Traumatologia da Faculdade de Ciências Médicas da Santa Casa de São Paulo, no período de 1994 a 2006. Este projeto de pesquisa foi avaliado e aprovado pelo Comitê de Ética em Pesquisa deste serviço (Projeto no163/07).

Os critérios de exclusão foram: fratura em mais de um nível, portadores de déficit neurológico prévio e fraturas patológicas ou provocadas por arma de fogo.

O tratamento baseou-se no uso de órtese tipo TLSO (Jewett) ou gesso em hiperextensão, mantido por quatro a seis meses, acompanhado de deambulação precoce.

Os pacientes nos quais se optou pelo tratamento conservador foram aqueles sem comprometimento neurológico ou critérios de instabilidade à radiografia simples, como perda de altura anterior do corpo vertebral maior que 50\% ou cifose localizada maior que 20 graus $^{31}$.

Os pacientes foram convocados por telefone ou carta registrada, submetidos ao exame clínico, categorizados conforme a escala de Frankel $^{32}$ e submetidos a questionários específicos e de qualidade de vida (visual-analógico de dor ou EVA ${ }^{33}$, Denis ${ }^{2}$, Oswestry ${ }^{34,35}$ e SF-36 $6^{36}$ )

A escala visual-analógica da dor (EVA) consiste em uma linha de $10 \mathrm{~cm}$ com os extremos fechados e à esquerda apresenta a indicação "sem dor" e à direita "dor insuportável". Cada paciente é orientado a marcar com um traço vertical o ponto que melhor corresponde à intensidade da dor no momento da avaliação e a determinação do resultado se faz medindo, em milímetros, a distância entre o ponto "sem dor" até a marca feita pelo paciente ${ }^{33}$.

A escala de dor e incapacidade de Denis caracteriza a dor em cinco tipos de acordo com a intensidade, falta ao trabalho e uso de medicações e a capacidade de retorno ao trabalho em cinco tipos, variando do retorno ao trabalho pesado à incapacidade de trabalhar².
O índice de Oswestry (Oswestry Disability Index) avalia a dor quanto à intensidade, cuidados pessoais, capacidade de erguer peso, caminhar, sentar, ficar em pé, vida sexual e viajar ${ }^{34,35}$. São atribuídos valores entre 0 e 5 a cada quesito, sendo o escore total dividido por 50 e valor final em percentagem.

O questionário SF-36 (The 36-item Short Form Health Survey Questionnaire) é instrumento genérico para a avaliação da qualidade de vida amplamente utilizado em diversas condições de saúde tendo sido traduzido para o português, adaptado culturalmente e validado para a população brasileira ${ }^{36}$. É composto por 36 questões, distribuídas em oito domínios: capacidade funcional, aspectos físicos, dor, estado geral de saúde, vitalidade, aspectos sociais, aspectos emocionais e saúde mental. A avaliação de cada item é feita utilizando-se o método dos pontos somados (método de Likert $^{37}$ ), com valores que variam de 0 a 100 pontos, sendo que os maiores índices indicam melhor qualidade de vida. Os índices dos domínios são obtidos a partir das pontuações dos itens de cada domínio, assim como os índices dos componentes derivam dos domínios a eles relacionados ${ }^{36}$.

Com base em modelo multidimensional de saúde, o SF-36 pode ser dividido em dois componentes: físico e mental. O componente físico é formado pelos domínios capacidade funcional (com dez itens ou questões), estado geral da saúde (com cinco itens), dor (com dois itens) e aspectos físicos (com quatro itens).

O componente mental é constituído dos domínios saúde mental (com cinco itens), vitalidade (com quatro itens), aspectos sociais (com dois itens) e aspectos emocionais (com três itens) ${ }^{36}$.

A porcentagem de comprometimento do canal vertebral foi mensurado nos cortes axiais da tomografia computadorizada de janela óssea mediante uso de régua milimetrada, segundo o método proposto por Trafton e Boyd ${ }^{38}$.

\section{Análise estatística}

Para a análise dos dados foi utilizado o programa SPSS (Statistical Package for Social Sciences), em sua versão 13.0. Foi aplicada a Análise de Correlação de Spearman, para verificarmos a correlação entre os resultados clínicos e a porcentagem de estreitamento do canal vertebral. Os dados foram comparados a resultados obtidos em outros trabalhos por intervalo de confiança, podendo ser similares (dentro do intervalo de confiança) ou diferentes (fora do intervalo de confiança).

Adotamos o nível de significância de 5\%, para a aplicação dos testes estatísticos, ou seja, quando a significância calculada (p) for menor do que 5\% $(0,050)$, encontramos uma diferença (ou relação) estatisticamente significante; quando a significância calculada (p) for igual ou maior do que $5 \%(0,050)$, encontramos uma diferença (ou relação) estatisticamente não-significante, ou seja, uma semelhança. 


\section{RESULTADOS}

Foram incluídos no trabalho 35 pacientes, sendo 15 do sexo masculino e 15 do feminino, com média de idade de $45 \pm 13$ anos, variando de 20 a 62 anos. O mecanismo de trauma foi a queda de altura em 22 pacientes $(73,3 \%)$, acidente automobilístico em $6(20 \%)$ e atropelamento ou trauma direto em 2 (6,67\%). A vértebra acometida foi L1 em 16 pacientes (53,3\%), T12 em 7 (23,3\%) e L2 em 7 (23,3\%) (Tabela 1). Constatamos 17 fraturas tipo A3.1 (56,6\%), 8 tipo A3.2 (26,7\%) e 5 tipo A3.3 (16,7\%). A porcentagem média de estenose do canal vertebral foi de $25 \pm 12 \%$, variando de 9 a 50\%. O tempo de internação hospitalar médio foi de $5 \pm 1,6$ dias, variando de quatro a dez dias e o tempo de seguimento médio foi de $95 \pm 39$ meses, variando de 36 a 145 meses. Nenhum paciente desenvolveu déficit neurológico durante o seguimento (Tabela 1).

A média da EVA31 foi de $3,1 \pm 3,1 \mathrm{~cm}$, variando de zero a $9 \mathrm{~cm}$, com intervalo de confiança de 1,99 a 4,21 cm (Tabela 2). Na análise da escala de dor de Denis ${ }^{2}$, encontramos dez pacientes $(33,3 \%)$ sem dor (P1), sete $(23,3 \%)$ com dor mínima e sem uso de medicação (P2), quatro $(13,3 \%)$ com dor moderada e sem interrupção do trabalho (P3), seis $(20 \%)$ com dor moderada ou intensa, uso frequente de medicação e ausência ocasional ao trabalho (P4) e três pacientes $(10 \%)$ com dor constante, incapacitante e uso crônico de medicação (P5) (Tabela 2). Por meio da escala de trabalho de Denis2, encontramos quatro pacientes $(13,4 \%)$ que retornaram ao trabalho pesado (W1), 11 (36,67\%) que retornaram ao trabalho sedentário ou pesado, mas com restrições (W2), seis $(20 \%)$ que mudaram de atividade (W3) e nove (30\%) incapazes para o trabalho (W5) (Tabela 2). A média do índice de Oswestry ${ }^{34}$ encontrada foi de $22 \% \pm 18,47$ (Tabela 2).

Os valores médios dos domínios do SF3636 foram comparados com os obtidos em outras publicações e destacados quando houve diferença estatisticamente significativa (Tabela 3).

Não houve correlação entre o estreitamento canal vertebral e os índices da EVA $(\mathrm{CC}=$ $0,31, \mathrm{p}<0,05)$ ou a Escala de Dor de Denis $(\mathrm{CC}=-0,35, \mathrm{p}>0,05)$. Houve correlação negativa entre a porcentagem de estenose do canal vertebral e a Escala de Trabalho de Denis, indicando que quanto menor foi a porcentagem de estenose do canal, maior foi o escore obtido na escala e, portanto, menor taxa de retorno ao trabalho $(\mathrm{CC}=-0,51, \mathrm{p}<0,05)$. Também houve correlação negativa entre o índice de Oswestry e a porcentagem de estenose do canal vertebral, indicando que os pacientes com maiores

\section{TABELA 1 - Resultados gerais}

\begin{tabular}{|c|c|c|c|c|c|c|}
\hline Paciente & Sexo & Idade & Nível & $\begin{array}{c}\text { Classificação } \\
\text { AO* }^{*}\end{array}$ & $\begin{array}{c}\text { Estenose } \\
(\%)^{* *}\end{array}$ & $\begin{array}{l}\text { Tempo de } \\
\text { Seguimento }\end{array}$ \\
\hline 1 & $\mathrm{~F}$ & 52 & Ll & A3.2 & 12,5 & 140 \\
\hline 2 & $M$ & 46 & LI & A3. 1 & 18 & 65 \\
\hline 3 & $\mathrm{~F}$ & 58 & $\mathrm{~T} 12$ & A3.3 & 12,5 & 60 \\
\hline 4 & $M$ & 51 & $\mathrm{~T} 12$ & A3.1 & 30 & 132 \\
\hline 5 & $M$ & 51 & LI & A3. 1 & 11 & 120 \\
\hline 6 & $M$ & 23 & L1 & A3.2 & 33 & 144 \\
\hline 7 & $\mathrm{~F}$ & 60 & L1 & A3.1 & 9 & 72 \\
\hline 8 & $M$ & 22 & L2 & A3.2 & 41 & 81 \\
\hline 9 & $\mathrm{~F}$ & 57 & $\mathrm{~T} 12$ & A3. 1 & 28,5 & 85 \\
\hline 10 & $M$ & 56 & L2 & A3.1 & 50 & 50 \\
\hline 11 & $\mathrm{~F}$ & 36 & L1 & A3.1 & 23 & 119 \\
\hline 12 & $M$ & 36 & L2 & A3. 1 & 11 & 37 \\
\hline 13 & $\mathrm{~F}$ & 20 & L 1 & A3.2 & 20 & 109 \\
\hline 14 & $\mathrm{~F}$ & 49 & $\mathrm{~T} 12$ & A3.2 & 13 & 140 \\
\hline 15 & $\mathrm{~F}$ & 31 & $\mathrm{~T} 12$ & A3.3 & 10 & 100 \\
\hline 16 & $\mathrm{~F}$ & 51 & L2 & A3.2 & 18 & 140 \\
\hline 17 & M & 53 & L2 & A3.3 & 30 & 108 \\
\hline 18 & $\mathrm{~F}$ & 20 & L1 & A3. 1 & 20 & 127 \\
\hline 19 & $M$ & 49 & L2 & A3.2 & 33,2 & 101 \\
\hline 20 & $\mathrm{~F}$ & 62 & L2 & A3.3 & 30 & 36 \\
\hline 21 & $\mathrm{~F}$ & 58 & Ll & A3. 1 & 22 & 87 \\
\hline 22 & $\mathrm{~F}$ & 56 & $\mathrm{~T} 12$ & A3. 1 & 17 & 36 \\
\hline 23 & $\mathrm{~F}$ & 38 & L1 & A3.2 & 50 & 143 \\
\hline 24 & $M$ & 58 & L1 & A3. 1 & 20 & 39 \\
\hline 25 & $M$ & 36 & L1 & A3.1 & 30 & 41 \\
\hline 26 & M & 50 & Ll & A3. 1 & 37,5 & 67 \\
\hline 27 & $M$ & 59 & LI & A3.3 & 23 & 117 \\
\hline 28 & M & 37 & L1 & A3.1 & 30 & 61 \\
\hline 29 & $\mathrm{~F}$ & 31 & L1 & A3.1 & 44 & 144 \\
\hline 30 & $M$ & 44 & T12 & A3. 1 & 25 & 145 \\
\hline
\end{tabular}

Magerl F, Aebi M, Gertzbein SD, Harms J, Nazarian S. A comprehensive classification of thoracic and lumbar injuries. Eur Spine J. 1994; 3(4): 184-201.

"Trafton PG, Boyd CA Jr. Computed tomography of thoracic and lumbar spine injuries. J Trauma. $1984 ; 24(6): 506-15$. 
TABELA 2 - Resultados clínicos

\begin{tabular}{clccc}
\hline Paciente & EVA $^{*}$ & Dor - Denis & Trabalho - Denis** & Oswestry** \\
\hline 1 & 0 & P1 & P5 & 4 \\
2 & 9 & P5 & P1 & 30 \\
3 & 6,2 & P4 & P5 & 26 \\
4 & 6,4 & P4 & P3 & 18 \\
5 & 4,7 & P4 & P5 & 22 \\
6 & 1,7 & P2 & P2 & 0 \\
7 & 7,5 & P3 & P3 & 16 \\
8 & 0,1 & P1 & P2 & 2 \\
9 & 0,6 & P3 & P2 & 18 \\
10 & 1,3 & P2 & P1 & 2 \\
11 & 8,7 & P5 & P5 & 25 \\
12 & 2,7 & P2 & P3 & 6 \\
13 & 4,9 & P3 & P2 & 6 \\
14 & 0,4 & P1 & P3 & 0 \\
15 & 6 & P4 & P5 & 22 \\
16 & 8 & P5 & P3 & 12 \\
17 & 0 & P1 & P1 & 3 \\
18 & 1 & P1 & P5 & 4 \\
19 & 3 & P1 & P2 & 6 \\
20 & 0 & P2 & P2 & 6 \\
21 & 7 & P4 & P5 & 19 \\
22 & 0 & P2 & P3 & 18 \\
23 & 2 & P2 & P5 & 12 \\
24 & 1,7 & P2 & P2 & 8 \\
25 & 0 & P1 & P2 & 0 \\
26 & 2,5 & P1 & P2 & 3 \\
27 & 6,7 & P4 & P5 & 22 \\
28 & 0,3 & P1 & P1 & 1 \\
29 & 0,6 & P3 & P2 & 18 \\
30 & 0,1 & P1 & P2 & 2 \\
\hline
\end{tabular}

"Million R, Hall W, Nilsen KH, Baker RD, Jayson MI. Assessment of the progress of the back pain patient. 1981 Volvo Award in Clinical Science. Spine. 1982; 7:204-12.

"Denis F, Armstrong GW, Searls K, Matta L. Acute thoracolumbar burst fractures in

the absence of neurologic deficit. A comparison between operative and nonoperative treatment. Clin Orthop Relat Res, 1984; Oct;(189): 142-9.

${ }^{* * *}$ Fairbank JCT, Pynsent PB. The Oswestry Disability Index. Spine 2000; 25:2940-53.

TABELA 3 - SF-36 e comparações

\begin{tabular}{|c|c|c|c|c|}
\hline Domínio & Média(DP) & $\begin{array}{l}\text { SF-36 média da } \\
\text { população americana } 1998^{*}\end{array}$ & $\begin{array}{l}\text { SF-36 } \\
\text { trabalho de Wood }{ }^{*}\end{array}$ & $\begin{array}{l}\text { SF-36 } \\
\text { trabalho de Vigato** }\end{array}$ \\
\hline Capacidade Funcional & $66,83(28,18)$ & $83,00 \S$ & $86,00 \S$ & 61,1 \\
\hline Limitação por Aspectos Físicos & $60,83(38,66)$ & $77,90^{\S}$ & $85,00 \S$ & $46,3^{\S}$ \\
\hline Dor & $64,1(27,27)$ & 70,20 & 72,00 & $47,3^{\S}$ \\
\hline Estado Geral de Saúde & $75,58(25,1)$ & 70,10 & 72,00 & $64 \S$ \\
\hline Vitalidade & $65,33(23,89)$ & 57,00 & $56,00 \$$ & $52,2^{\S}$ \\
\hline Aspectos Sociais & $78,5(24,46)$ & 83,60 & 84,00 & $66,1^{\S}$ \\
\hline Limitação por Aspectos Emocionais & $60(41,43)$ & $83,10^{\S}$ & $79,00 \S$ & 48,1 \\
\hline Saúde Mental & $70,8(23,42)$ & 75,20 & 75,00 & 65,4 \\
\hline Componente físico & $66,84(26,65)$ & 75,3 & $78,75^{\S}$ & \\
\hline Componente mental & $68,66(25)$ & 74,73 & 73,50 & \\
\hline
\end{tabular}

Ware JE, Kosinski M, Gandek BG, et al. The factor structure and content of the SF-36 (IQOLA) Project. J Clin Epidemiol. 1998;51 (1 1): $1159-65$. "Wood K, Buttermann G, Mehbod A, et al. Operative compared with nonoperative treatment of a thoracolumbar burst fracture without neurological deficit. A prospective, randomized study. J Bone Joint Surg Am. 2003;85-A(5):773-81. Erratum in: J Bone Joint Surg Am. 2004; 86-A(6):1283.

"'Vigatto R, Alexandre NM, Correa Filho HR. Development of a Brazilian Portuguese version of the Oswestry Disability Index: cross-cultural adaptation, reliability, and validity. Spine. 2007; 32(4):481-6.

$\S$ Valores fora do intervalo de confiança ou diferença estatisticamente significativa 


\section{TABELA 4 - Correlações com o grau de estreitamento do canal vertebral}

\begin{tabular}{|c|c|c|}
\hline & $\begin{array}{l}\text { Coeficiente de } \\
\text { correlação }(\mathrm{CC})^{\S}\end{array}$ & $\begin{array}{l}\text { Significância } \\
\text { (p) }{ }^{\S \S}\end{array}$ \\
\hline $\mathrm{EVA}^{*}$ & $-0,31$ & 0,091 \\
\hline \multicolumn{3}{|l|}{ Escala de Denis* $^{* *}$} \\
\hline Dor & $-0,35$ & 0,065 \\
\hline Trabalho & $-0,51^{\S}$ & $0,004 \S$ \\
\hline Índice de Oswestry ${ }^{* * *}$ & $-0,40 \S$ & $0,027 \S$ \\
\hline Domínio "D" & $-0,18$ & 0,353 \\
\hline \multicolumn{3}{|l|}{ ou "caminhar" } \\
\hline \multicolumn{3}{|l|}{ SF-36 $6^{* * * *}$} \\
\hline Capacidade & $0,46^{\S}$ & $0,011 \S$ \\
\hline \multicolumn{3}{|l|}{ Funcional } \\
\hline Limitação física & 0,33 & 0,073 \\
\hline Dor & 0,32 & 0,081 \\
\hline Estado geral & 0,22 & 0,237 \\
\hline \multicolumn{3}{|l|}{ de saúde } \\
\hline Vitalidade & 0,12 & 0,542 \\
\hline Aspectos sociais & 0,07 & 0,695 \\
\hline Aspectos emocionais & 0,32 & 0,089 \\
\hline Saúde Mental & 0,12 & 0,440 \\
\hline Componente físico & $0,36 \S$ & $0,049^{*}$ \\
\hline Componente mental & 0,20 & 0,280 \\
\hline
\end{tabular}

"Million R, Hall W, Nilsen KH, Baker RD, Jayson Ml. Assessment of the progress of the back pain patient. 1981 Volvo Award in Clinical Science. Spine. 1982; 7:204-12.

"Denis F, Armstrong GW, Searls K, Matta L. Acute thoracolumbar burst fractures in the absence of neurologic deficit. A comparison between operative and nonoperative treatment. Clin Orthop Relat Res, 1984; Oct:(189): 1 42-9.

"'Fairbank JCT, Pynsent PB. The Oswestry Disability Index. Spine 2000; 25:2940-53.

... Vigatto R, Alexandre NM, Correa Filho HR. Development of a Brazilian Portuguese version of the Oswestry Disability Index: cross-cultural adaptation, reliability, and validity. Spine. 2007; 32(4):481-6.

${ }^{\S}$ Coeficiente de correlação obtido pelo teste de Spearmann.

\$§Significância estatística ou $p<0,05$.

Quando analisamos os domínios expressos em apenas dois componentes, notamos correlação entre o componente físico e a porcentagem de estreitamento do canal vertebral, demonstrando que os pacientes com maiores índices (menor incapacidade física) apresentavam maior porcentagem de estreitamento do canal vertebral $(\mathrm{CC}=0,36, \mathrm{p}<0,05)$ (Tabela 4).

\section{Caso Clínico}

As imagens ao lado referem-se ao paciente número de ordem 6. Figuras 1 a 4.
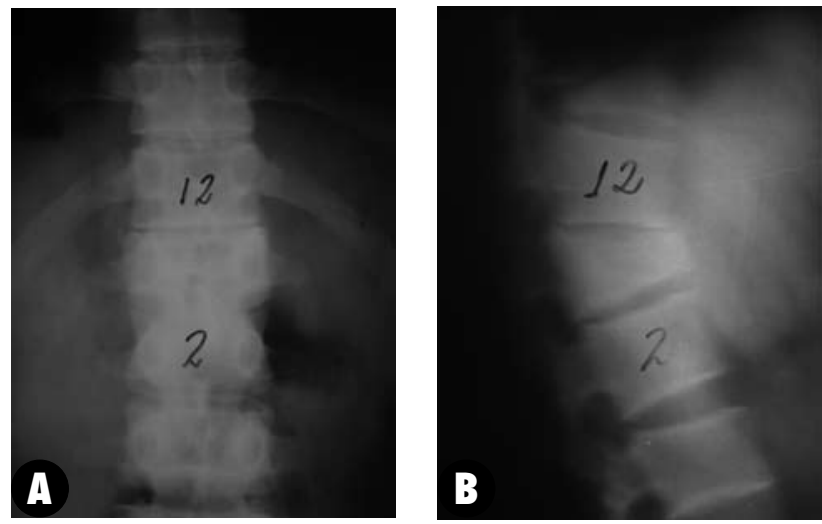

Figura 1

(A) e (B) - Radiografias iniciais

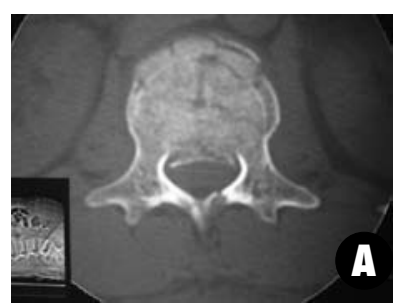

Figura 2

(A) e (B) - Cortes tomográficos
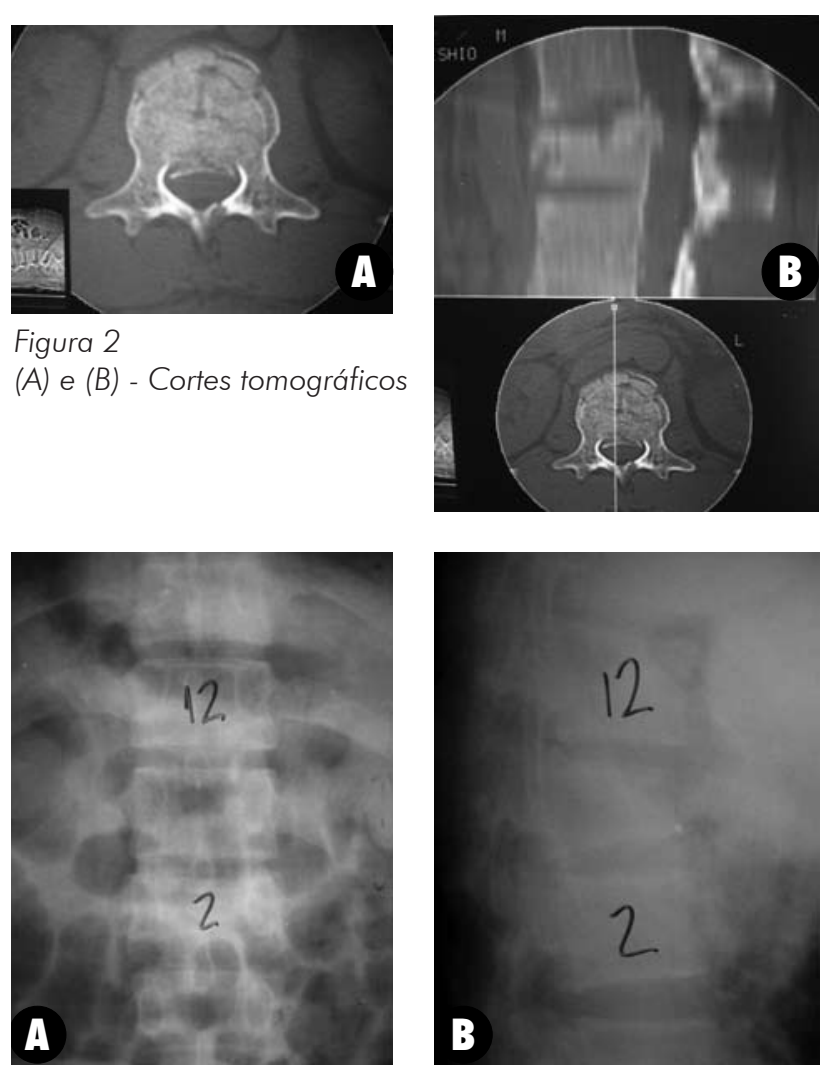

Figura 3

(A) e (B) - Controle após a confecção de colete gessado
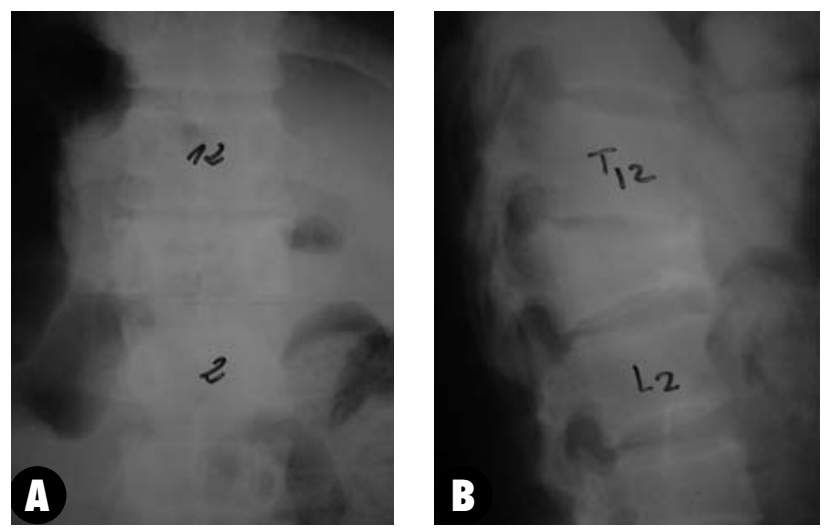

Figura 4

(A) e (B) - Radiografias com 36 meses de seguimento 


\section{DISCUSSÃO}

O tratamento da fratura toracolombar explosão ainda é tema de debate e motivo para muitos trabalhos na literatura. No entanto, notamos a falta de trabalhos que avaliem desfechos clínicos de tratamento mediante a utilização de escalas clínicas e questionários de qualidade de vida. Em nosso trabalho, procuramos avaliar os resultados por meio de índices amplamente aceitos e que revelam as limitações físicas e psíquicas decorrentes do tratamento empregado $2,33,34,35,36$.

Wood et al.39 avaliaram comparativamente os resultados do tratamento conservador e cirúrgico em fraturas explosão toracolombares e obtiveram, por meio da escala análogo-visual da dor (EVA), valores de 1,9 e 3,3cm, respectivamente em um período de seguimento de 46 e 43 meses. Em nosso trabalho, obtivemos um valor médio de $3,1 \mathrm{~cm}$, sendo semelhante ao grupo cirúrgico do trabalho referido, porém, o nosso período de seguimento médio foi cerca de duas vezes superior (95 meses), podendo demonstrar o comportamento dos resultados a longo prazo do tratamento conservador.

A escala de dor de Denis revela que a maioria dos pacientes $(70 \%)$ não se encontram limitados pela dor, mantendo a capacidade de trabalho. No entanto, houve uma elevada taxa de pacientes que não retornaram ao trabalho $(30 \%)$, sendo que estes pacientes apresentavam menor porcentagem de estreitamento do canal vertebral. Em nosso país, devido à quantidade de pacientes aptos às suas funções que recorrem ao auxíliodoença, a taxa de retorno ao trabalho não representa parâmetro fidedigno para a avaliação de resultados clínicos, podendo comportar-se como fator de confusão.

A média do índice de Oswestry encontrado em nossa série foi de $22 \%$, valor comparativamente superior ao descrito por Wood et al. ${ }^{39}$ para os pacientes tratados de forma conservadora $(11 \%)$ e similar ao grupo tratado cirurgicamente $(21 \%)$. Ao compararmos ao descrito para pacientes portadores de lombalgia em trabalhos realizados em nosso país ${ }^{35}$, notamos valores semelhantes $(25 \%)$. Na análise do domínio responsável pela distância caminhada no índice de Oswestry (domínio D), não houve correlação significativa, indicando que os pacientes com maior estreitamento do canal vertebral apresentaram distâncias caminhadas semelhantes aos com menor estreitamento.

A comparação dos resultados por intervalo de confiança mostra que os valores obtidos nos domínios Capacidade Funcional, Limitação por Aspectos Físicos e Limitação por Aspectos Emocionais foram inferiores ao padrão da população norteamericana em 1998, porém a análise dos domínios sumarizados em componentes físico e mental não demonstraram diferença significativa ${ }^{40}$. Ao compararmos os resultados do questionário SF-36 aos obtidos por Wood et al. ${ }^{39}$, obtivemos valores inferiores nos domínios Capacidade Funcional, Limitação por Aspectos Físicos e Limitação por Aspectos Emocionais, porém superiores no domínio Vitalidade. Ao analisarmos o questionário sintetizado em dois componentes, obtivemos valores inferiores no Componente Físico. Ao compararmos ao trabalho de Vigato et al. ${ }^{35}$, em que foram avaliados pacientes portadores de lombalgia crônica, obtivemos valores superiores quantos aos domínios Limitação por Aspectos Físicos, Dor, Estado Geral de Saúde, Vitalidade e Aspectos Sociais, confirmando os bons resultados obtidos em nossa amostra. A porcentagem de estreitamento do canal vertebral tem sido utilizada como parâmetro de indicação cirúrgica, mas a sua significância em pacientes sem comprometimento neurológico permanece incerta.

Diversos estudos não encontraram correlação entre a porcentagem de estreitamento do canal vertebral e os resultados clínicos $^{17-21}$. Da mesma forma, não encontramos correlação entre o estreitamento do canal vertebral inicial e os índices da EVA ou a Escala de Dor de Denis. Porém, os pacientes que não retornaram ao trabalho apresentaram menor grau de estreitamento do canal vertebral.

Diversos estudos demonstraram que ocorre remodelação espontânea do canal vertebral após fratura explosão toracolombar, especialmente durante o primeiro ano após a fratura, sendo maior em pacientes com maior porcentagem de estreitamento ${ }^{22-28}$. Encontramos também correlação negativa do índice de Oswestry com a porcentagem de estreitamento do canal vertebral, demonstrando que os pacientes com menores índices de Oswestry, ou seja, melhor condição clínica, apresentaram maior porcentagem de estreitamento do canal vertebral. $\mathrm{Na}$ análise do SF-36, encontramos situação semelhante, sendo que os pacientes com maior estreitamento do canal vertebral apresentaram melhor capacidade funcional. Os resultados obtidos confirmam a inexistência de correlação entre maior estreitamento do canal vertebral e piores resultados clínicos, conforme descrito na literatura.

\section{CONCLUSÃO}

Em nosso estudo não observamos correlação entre o estreitamento do canal vertebral e os resultados clínicos do tratamento conservador em pacientes com fratura toracolombar explosão.

\section{REFERÊNCIAS}

1. Singh K, Kim D, Vaccaro AR. Thoracic and Lumbar Spinal Injuries. In: RothmanSimeone The Spine. Philadelphia: Saunders Elsevier; 2006. p.1132-3.

2. Denis F, Armstrong GW, Searls K, Matta L. Acute thoracolumbar burst fractures in the absence of neurologic deficit. A comparison between operative and nonoperative treatment. Clin Orthop Relat Res. 1984;(189):142-9.
3. McEvoy RD,Bradford DS. The management of burst fractures of the thoracic and lumbar spine. Experience in 53 patients. Spine 1985; 10(7):631-7.

4. Krompinger WJ, Fredrickson BE, Mino DE, Yuan HA. Conservative treatment of fractures of the thoracic and lumbar spine. Orthop Clin North Am. 1986; 17(1):16170 .
5. Weinstein JN, Collalto P, Lehmann TR. Thoracolumbar "burst" fractures treated conservatively: a long-term follow-up. Spine. 1988; 13(1):33-8.

6. Knight RQ, Stornelli DP, Chan DP, Devanny JR, Jackson KV. Comparison of operative versus nonoperative treatment of lumbar burst fractures. Clin Orthop Relat Res, 1993;(293):112-21. 
7. Connolly JF. Differences between operative and nonoperative treatment of lumbar burst fractures. Clin Orthop Relat Res. 1994; (306):286.

8. Shen WJ, Liu TJ, Shen YS. Nonoperative treatment versus posterior fixation for thoracolumbar junction burst fractures without neurologic deficit. Spine. 2001;26(9):1038-45.

9. Thomas KC, Bailey CS, Dvorak MF, Kwon B, Fisher C. Comparison of operative and nonoperative treatment for thoracolumbar burst fractures in patients without neurological deficit: a systematic review. J Neurosurg Spine. 2006; 4(5):351-8.

10. Yi L, Jingping B, Gele J, Baoleri X, Taixiang W. Operative versus nonoperative treatment for thoracolumbar burst fractures without neurological deficit. Cochrane Database Syst Rev. 2006 Oct 18;(4):CD005079.

11. Kim NH, Lee HM, Chun IM. Neurologic injury and recovery in patients with burst fracture of the thoracolumbar spine. Spine. 1999; 24(3):290-3.

12. Vaccaro AR, Nachwalter RS, Klein GR, Sewards JM, Albert TJ, Garfin SR. The significance of thoracolumbar spinal canal size in spinal cord injury patients. Spine. 2001;26(4):371-6.

13. Crossman PT, Scott JM. Does 'canal clearance' affect neurological outcome after thoracolumbar burst fractures? J Bone Joint Surg Br. $2001 ; 83(1): 151-2$.

14. Mohanty SP, Venkatram N. Does neurological recovery in thoracolumbar and lumbar burst fractures depend on the extent of canal compromise? Spinal Cord. 2002;40(6):295-9.

15. Meves R, Avanzi O. Correlation among canal compromise, neurologic deficit, and injury severity in thoracolumbar burst fractures. Spine. 2006;31(18):2137-41.

16. Dai LY, Wang XY, Jiang LS. Neurologic recovery from thoracolumbar burst fractures: is it predicted by the amount of initial canal encroachment and kyphotic deformity? Surg Neurol. 2007;67(3):232-7.

17. McAfee PC, Yuan HA, Fredrickson BE, Lubicky JP. The value of computed tomography in thoracolumbar fractures. An analysis of one hundred consecutive cases and a new classification. J Bone Joint Surg Am. 1983;65(4):461-73.

18. Esses SI. The placement and treatment of thoracolumbar spine fractures. An algorithmic approach. Orthop Rev. 1988;17(6):571-84.

19. Reid DC, Hu R, Davis LA, Saboe LA. The nonoperative treatment of burst fractures of the thoracolumbar junction. J Trauma. 1988;28(8):1188-94.

20. Shen WJ, Shen YS. Nonsurgical treatment of three-column thoracolumbar junction burst fractures without neurologic deficit. Spine. 1999;24:412-5.

21. Kinoshita H, Nagata Y, Ueda H, Kishi K. Conservative treatment of burst fractures of the thoracolumbar and lumbar spine. Paraplegia. 1993;31(1):58-67.
22. Johnsson R, Herrlin K, Hägglund G, Strömqvist B. Spinal canal remodeling after thoracolumbar fractures with intraspinal bone fragments. 17 cases followed 1-4 years. Acta Orthop Scand. 1991; 62(2):125-7.

23. Scapinelli R, Candiotto S. Spontaneous remodeling of the spinal canal after burst fractures of the low thoracic and lumbar region. J Spinal Disord. 1995; 8(6):486-93.

24. Yazici M, Atilla B, Tepe S, Calisir A. Spinal canal remodeling in burst fractures of the thoracolumbar spine: a computerized tomographic comparison between operative and nonoperative treatment. J Spinal Disord. 1996;9(5):409-13.

25. Karlsson MK, Hasserius R, Sundgren P, Redlund-Johnell I, Ohlin A. Remodeling of the spinal canal deformed by trauma. J Spinal Disord. 1997; 10(2):157-61.

26. de Klerk LW, Fontijne WP, Stijnen T, Braakman R, Tanghe HL, van Linge B. Spontaneous remodeling of the spinal canal after conservative management of thoracolumbar burst fractures. Spine. 1998; 23(9):1057-60.

27. Dai LY. Remodeling of the spinal canal after thoracolumbar burst fractures. Clin Orthop Relat Res. 2001;(382):119-23.

28. Mumford J, Weinstein JN, Spratt KF, Goel VK. Thoracolumbar burst fractures. The clinical efficacy and outcome of nonoperative management. Spine. 1993; 18(8):955-70.

29. Fontijine W, De Kleak L, Braakman R, et al. CT scan predicton of neurological deficit in thoracolumbar burst fractures. J Bone Joint Surg Br.1992;75(5): 683-5. Erratum in: J Bone Joint Surg Br 1993.

30. Magerl F, Aebi M, Gertzbein SD, Harms J, Nazarian S. A comprehensive classification of thoracic and lumbar injuries. Eur Spine J. 1994; 3(4):184-201.

31. Cantor JB, Lebwohl NH, Garvey T, Eismont FJ. Nonoperative management of stable thoracolumbar burst fractures with early ambulation and bracing. Spine 1993;18(8):971-6.

32. Frankel HL, Hancock DO, Hyslop G, et al. The value of postural reduction in the initial management of closed injuries of the spine with paraplegia and tetraplegia. Paraplegia. 1969; 7(3):179-92.

33. Million R, Hall W, Nilsen KH, Baker RD, Jayson MI. Assessment of the progress of the back pain patient. 1981 Volvo Award in Clinical Science. Spine. 1982; 7(3):204-12.
34. Fairbank JCT, Pynsent PB. The Oswestry Disability Index. Spine 2000; 25(22):294053.

35. Vigatto R, Alexandre NM, Correa Filho HR. Development of a Brazilian Portuguese version of the Oswestry Disability Index: cross-cultural adaptation, reliability, and validity. Spine. 2007; 32(4):481-6.

36. Ciconelli RM. Tradução para o português e validação do questionário genérico de avaliação de qualidade de vida "medical outcomes study 36-item sort form health survey (SF36)" [tese ]. São Paulo: Universidade Federal de São Paulo; 1997.

37. Wolfle D, Likert R, et al. Standards for appraising psychological research. Am Psychol. 1949; 4(8):320-8.

38. Trafton PG, Boyd CA Jr. Computed tomography of thoracic and lumbar spine injuries. J Trauma. 1984; 24(6):506-15.

39. Wood K, Buttermann G, Mehbod A, et al. Operative compared with nonoperative treatment of a thoracolumbar burst fracture without neurological deficit. A prospective, randomized study. J Bone Joint Surg Am. 2003;85(5):773-81. Erratum in: J Bone Joint Surg Am. 2004; 86-A(6): 1283.

40. Ware JE, Kosinski M, Gandek BG, et al. The factor structure and content of the SF-36 Health Survey in 10 countries: Results from the International Quality of Life Assessment (IQOLA) Project. J Clin Epidemiol 1998;1159-65.

\section{Correspondência}

Osmar Avanzi

Rua Dr. Cesário Mota Júnior, 112, Santa

Cecília, São Paulo, SP, Brasil

CEP: 01277-900

E-mail: coluna@santacasasp.org.br 